\title{
Fungemia due to Lachancea fermentati: a case report
}

\author{
Anne-Marie Leuck*, Meghan K Rothenberger and Jaime S Green
}

\begin{abstract}
Background: Lachancea fermentati is an environmental yeast that is also used in the fermentation of alcoholic drinks. It has not previously been described as a human pathogen although the closely related yeast, Saccharomyces boulardii, can cause fungemia. Here we report a case of $L$. fermentati acting as a pathogen in a septic patient with cultures positive from blood, peritoneal fluid, bile, and sputum.

Case presentation: A 36 year-old Caucasian man was hospitalized with acute alcoholic hepatitis complicated by Escherichia coli spontaneous bacterial peritonitis. Three days after admission, he developed new fevers with sepsis requiring mechanical ventilation and vasopressor support. He was found to have a bowel perforation. Cultures from blood, peritoneal fluid, and sputum grew a difficult-to-identify yeast. Micafungin was started empirically. On hospital day 43 the yeast was identified as L. fermentati with low minimum inhibitory concentrations (by Epsilometer test) to all antifungals tested. Micafungin was changed to fluconazole to complete a 3-month course of therapy. Serial peritoneal fluid cultures remained positive for 31 days. One year after his initial hospitalization the patient had ongoing cirrhosis but had recovered from fungemia.

Conclusion: This case demonstrates the need for clinicians to consider host factors when interpreting culture results with normally non-pathogenic organisms. In this immunocompromised host L. fermentati caused disseminated disease. We believe his hobby of brewing alcohol led to colonization with $L$. fermentati, which then resulted in invasive disease when the opportunity arose.
\end{abstract}

Keywords: Lachancea fermentati, Fungemia, Opportunistic pathogen

\section{Background}

Non-Candida fungemia has become increasingly problematic among immunocompromised hosts, and fungal genera such as Trichosporon, Cryptococcus, Rhodotorula, Malassezia, and Blastoschizomyces have become recognized as opportunistic pathogens [1-3]. The genus Saccharomyces has also been described as a human pathogen [4-6]. We describe a case of fungemia and sepsis due to the yeast Lachancea fermentati, a species closely related to the saccharomycetes. The known potential of Saccharomyces to act as a pathogen suggests that the related species, L. fermentati, may also have the ability to cause disease in the appropriate clinical setting.

\footnotetext{
* Correspondence: leuck002@umn.edu

Department of Medicine, University of Minnesota, D-416 Mayo Building, MMC 250, 420 Delaware St. SE, Minneapolis, MN 55455, USA
}

\section{Case presentation}

A 36 year-old man with acute alcoholic hepatitis was hospitalized due to Escherichia coli peritonitis attributed to spontaneous bacterial peritonitis. His social history was notable for brewing his own alcohol and alcohol abuse. He initially improved symptomatically, but three days after admission he developed a fever to $38.1^{\circ} \mathrm{C}$ and multi-organ failure requiring mechanical ventilation, continuous renal replacement therapy, and vasopressor support. He clinically stabilized with intensive critical care support and initiation of empiric antibiotics (vancomycin, meropenem, and micafungin). Blood cultures at the time of admission were negative. One of four blood culture bottles drawn at the time of clinical decompensation became positive for yeast after 12 hours of incubation (BacT Alert System', Biomerieux, Durham, NC). Sputum and peritoneal fluid cultures collected the following day also grew the same yeast. Culture showed creamy white colonies on Sabouroud-dextrose agar 
(Figure 1). The yeast was not immediately identifiable by the clinical microbiology lab.

During the two-month admission, he was found to have loculated ascites associated with a fistulous connection to bowel. Multiple abdominal abscess and peritoneal fluid cultures had polymicrobial growth (Table 1). Despite the intermittent presence of other organisms, every peritoneal culture over a one-month period, nine in total, grew the same difficult-to-identify yeast. This same yeast also grew from three sputum cultures (Table 1). The yeast isolated from sputum was felt to more likely represent colonization than infection. The patient had pleural effusions attributed to his ascites but he did not have a clinical pneumonia. Urine cultures remained negative. Medical management included aggressive drainage of the abdominal fluid collections and continuation of broad-spectrum antimicrobials including meropenem, vancomcyin, and micafungin. Over time, the patient slowly improved with resolution of the abdominal pain, fever, leukocytosis, and abdominal abscesses.

On hospital day 43, the yeast isolated from blood, peritoneal fluid, bile, and sputum was identified as Lachancea fermentati by the Fungus Testing Laboratory at the University of Texas Health Science Center. Identification was based on cornmeal agar morphology, cycloheximide susceptibility studies, temperature studies, and sequencing of the D1/D2 regions of 28 s rDNA. No susceptibility breakpoints are available for L. fermentati, but the minimum inhibitory concentration (MIC) was low for all antifungal agents tested (Table 2). When MIC values were known, micafungin was changed to fluconazole $100 \mathrm{mg}$ daily (approximately $3 \mathrm{mg} / \mathrm{kg}$, the renal-adjusted dose equivalent of $6 \mathrm{mg} / \mathrm{kg}$ ). He completed 6 weeks of therapy with micafungin and an additional 6 weeks of fluconazole, for a total 3 months of antifungal therapy. One year after his initial presentation he remained abstinent from alcohol and fully recovered from the fungemia. He does have cirrhosis and at the time of his last clinic visit he continued on ciprofloxacin for spontaneous bacterial peritonitis prophylaxis.

\section{Conclusions}

L. fermentati is an environmental, saprophytic yeast found in decaying material. In its natural setting, Lachancea colonizes leaf surfaces and may provide a natural buffer against plant pathogens [7]. Lachancea species, in combination with Saccharomyces cerevisiae, are commonly used in the fermentation process to make wine and cachaca (a drink made from fermented sugar) [8,9], and have been shown to enhance the quality and aroma of these beverages [10].

While L. fermentati has been recognized as a component of fermented drinks, it may be more pervasive than previously thought. L. fermentati was found in more than $50 \%$ of olive oil mills tested [5], and an investigation of commercially available drinks in Brazil also found L. fermentati in coconut juice and reconstituted fruit juices [11]. A species with a close evolutionary relationship to $L$. fermentati, L. thermotolerans, is present on the leaves of deciduous trees in the autumn when fruit would be harvested [7]. With this increasing recognition of Lachancea's environmental presence, clinicians and clinical microbiologists should be aware of its pathogenic potential.

Recent progress has also been made in the classification of Lachancea yeasts. L. fermentati, formerly known as Zygosaccharomyces fermentati, is related to the genus Saccharomyces. Prior to DNA sequencing, classification of these organisms was based on morphologic and phenotypic characteristics. The advent of nuclear-based rDNA sequencing technology, coupled with multigene-based phylogenetic analyses, has led to reclassification of the 11 clades of Saccharomycetaceae. The genus Lachancea consists of five species, L. cidri, L. fermentati, L. kluyveri, $L$. thermotolerans and L. waltii. The organism is further characterized by vegetative reproduction with multilateral

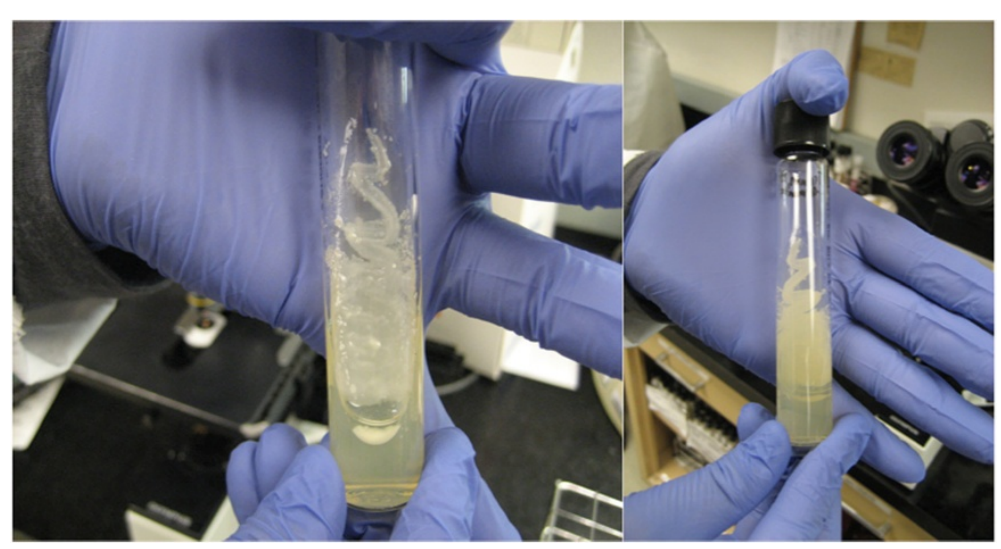

Figure 1 Front and back view of $L$. fermentati grown on Sabouroud dextrose agar showing the creamy white colonies typical of yeast. 
Table 1 Summary of positive cultures during hospitalization

\begin{tabular}{|c|c|c|}
\hline $\begin{array}{l}\text { Hospital day } \\
\text { of culture }^{a}\end{array}$ & Site of isolation & Organism(s) isolated \\
\hline Day 3 & Peritoneal fluid & Eggerthalla lenti \\
\hline Day 5 & Blood & Lachancea fermentati \\
\hline \multirow[t]{2}{*}{ Day 6} & Sputum & L. fermentati \\
\hline & Peritoneal fluid & $\begin{array}{l}\text { L. fermentati, Enterococcus spp., } \\
\text { Lactobacillus }\end{array}$ \\
\hline Day 8 & Sputum & L. fermentati, Candida rugosa \\
\hline Day 9 & Peritoneal fluid & L. fermentati \\
\hline Day 10 & Peritoneal fluid & L. fermentati \\
\hline Day 11 & Peritoneal fluid & L. fermentati \\
\hline Day 12 & Peritoneal fluid & L. fermentati \\
\hline \multirow[t]{2}{*}{ Day 15} & Peritoneal fluid & L. fermentati \\
\hline & Sputum & L. fermentati \\
\hline Day 16 & Bile & $\begin{array}{l}\text { L. fermentati, coagulase negative } \\
\text { staphylococcus }\end{array}$ \\
\hline Day 24 & Peritoneal fluid & L. fermentati \\
\hline Day 36 & Abdominal abscess & $\begin{array}{l}\text { L. fermentati, Candida parapsilosis, } \\
\text { Bacteroides spp., Pseudomonas } \\
\text { aeruginosa, Clostridium spp. }\end{array}$ \\
\hline Day 46 & Sputum & $\begin{array}{l}\text { C. parapsilosis, coagulase negative } \\
\text { staphylococcus }\end{array}$ \\
\hline Day 50 & Sputum & $\begin{array}{l}\text { C. parapsilosis, coagulase negative } \\
\text { staphylococcus }\end{array}$ \\
\hline
\end{tabular}

${ }^{a}$ Note that the patient also had a peritoneal fluid culture positive for Escherichia coli prior to admission.

budding on a narrow base and fermentation of glucose in addition to at least one other sugar [12]. Based on comparative analyses of rDNA sequences and molecular karyotyping of Lachancea species, the yeast appears to have eight chromosomes [13], and its preferred growing temperature is $25-37^{\circ} \mathrm{C}$ [14]. This range includes normal human body temperature and suggests that $L$. fermentati could act as a pathogen.

Of interest, one of this patient's early paracenteses grew Eggerthella lenta, which is associated with the gastrointestinal flora of wine drinkers [15]. This patient did indeed brew his own alcohol. Taken together, the presence of two microorganisms associated with fermented drinks suggests that his microbiome may have been

Table 2 Lachancea fermentati minimum inhibitory concentrations (MIC) by Epsilometer test (E-test)

\begin{tabular}{lc}
\hline Antifungal agent & MIC by E-test $(\mathbf{u g} / \mathbf{m L})$ \\
\hline Amphotericin B & 0.064 \\
Caspofungin & 0.38 \\
Fluconazole & 0.75 \\
Itraconazole & 0.064 \\
Voriconazole & 0.016 \\
\hline
\end{tabular}

affected by his alcohol brewing and consumption, resulting in infection with an unusual pathogen, L. fermentati. In a similar case, $S$. cerevisiae (baker's yeast) caused disseminated disease in a baker's wife with leukemia, which was thought to be associated with occupation-related colonization [16].

To the best of our knowledge, this is the first case reporting $L$. fermentati as a human pathogen. This case of $L$. fermentati fungemia highlights the ability of nonpathogenic microorganisms to cause disease in unusual situations depending on host risk factors and clinical circumstances. If this patient had not been fungemic and septic, this yeast might have been considered a colonizing species. However, in this immunocompromised host with a perforated viscus, it acted as a pathogen and required treatment.

\section{Consent}

Written informed consent was obtained from the patient for publication of this case report. A copy of the written consent is available for review by the Editor of this journal.

\section{Competing interests}

The authors declare that they have no competing interests.

\section{Authors' contributions}

AML wrote the manuscript. JG and MR contributed patient data and were involved in editing the manuscript. All authors read and approved the final manuscript.

\section{Acknowledgements}

We would like to thank Annette Fothergill at the Fungus Testing Laboratory at the University of Texas Health Science Center for identification of the yeast species. AML is supported by a National Institutes of Health Ruth L. Kirschstein National Research Service Award Institutional Research Training Grant [5T32AI055433-09]. JG and MR do not have grant support.

Received: 10 January 2014 Accepted: 1 May 2014

Published: 10 May 2014

\section{References}

1. Yamamoto M, Takakura S, Hotta G, Matsumura Y, Matsushima A, Nagao M, Ito $Y$, Ichiyama S: Clinical characteristics and risk factors of non-Candida fungaemia. BMC Infect Dis 2013, 13:247.

2. Colombo AL, Padovan AC, Chaves GM: Current knowledge of Trichosporon spp. and Trichosporonosis. Clin Microbio/ Rev 2011, 24(4):682-700.

3. Gaitanis $G$, Magiatis P, Hantschke M, Bassukas ID, Velegraki A: The Malassezia genus in skin and systemic diseases. Clin Microbiol Rev 2012, 25(1):106-141.

4. Thygesen JB, Glerup H, Tarp B: Saccharomyces boulardii fungemia caused by treatment with a probioticum. BMJ Case Rep 2012. doi:10.1136/ bcr.06.2011.4412

5. Hamoud S, Keidar Z, Hayek T: Recurrent Saccharomyces cerevisiae fungemia in an otherwise healthy patient. Isr Med Assoc J 2011, 13(9):575-576.

6. Cassone M, Serra P, Mondello F, Girolamo A, Scafetti S, Pistella E, Venditti M: Outbreak of Saccharomyces cerevisiae subtype boulardii fungemia in patients neighboring those treated with a probiotic preparation of the organism. J Clin Microbiol 2003, 41(11):5340-5343.

7. Slavikova E, Vadkertiova R, Vranova D: Yeasts colonizing the leaf surfaces. J Basic Microbiol 2007, 47(4):344-350.

8. Nova MX, Schuler AR, Brasileiro BT, Morais MA Jr: Yeast species involved in artisanal cachaca fermentation in three stills with different technological levels in Pernambuco, Brazil. Food Microbiol 2009, 26(5):460-466. 
9. Li SS, Cheng C, Li Z, Chen JY, Yan B, Han BZ, Reeves M: Yeast species associated with wine grapes in China. Int J Food Microbiol 2010, 138(1-2):85-90.

10. Comitini F, Gobbi M, Domizio P, Romani C, Lencioni L, Mannazzu I, Ciani M: Selected non-Saccharomyces wine yeasts in controlled multistarter fermentations with Saccharomyces cerevisiae. Food Microbiol 2011, 28(5):873-882.

11. Maciel NO, Pilo FB, Freitas LF, Gomes FC, Johann S, Nardi RM, Lachance MA, Rosa CA: The diversity and antifungal susceptibility of the yeasts isolated from coconut water and reconstituted fruit juices in Brazil. Int J Food Microbiol 2013, 160(3):201-205.

12. Kurtzman CP: Phylogenetic circumscription of Saccharomyces, Kluyveromyces and other members of the Saccharomycetaceae, and the proposal of the new genera Lachancea, Nakaseomyces, Naumovia, Vanderwaltozyma and Zygotorulaspora. FEMS Yeast Res 2003, 4(3):233-245.

13. Naumova ES, Serpova EV, Naumov GI: Molecular systematics of Lachancea yeasts. Biochemistry (Mosc) 2007, 72(12):1356-1362

14. Vuille C, Nidorf M, Weyman AE, Picard MH: Natural history of vegetations during successful medical treatment of endocarditis. Am Heart J 1994, 128(6 Pt 1):1200-1209.

15. Queipo-Ortuno MI, Boto-Ordonez M, Murri M, Gomez-Zumaquero JM, Clemente-Postigo M, Estruch R, Cardona Diaz F, Andres-Lacueva C, Tinahones FJ: Influence of red wine polyphenols and ethanol on the gut microbiota ecology and biochemical biomarkers. Am J Clin Nutr 2012, 95(6):1323-1334.

16. Choi G, Meijer SL, Hazenberg MD: Disseminated bread yeast fungaemia in a baker's wife with acute myeloid leukaemia. Br J Haematol 2012, 158(3):298.

doi:10.1186/1471-2334-14-250

Cite this article as: Leuck et al.: Fungemia due to Lachancea fermentati: a case report. BMC Infectious Diseases 2014 14:250.

\section{Submit your next manuscript to BioMed Central and take full advantage of:}

- Convenient online submission

- Thorough peer review

- No space constraints or color figure charges

- Immediate publication on acceptance

- Inclusion in PubMed, CAS, Scopus and Google Scholar

- Research which is freely available for redistribution 